Self-reported obstructive sleep apnea, simple snoring, and various markers of sleep-disordered breathing as predictors of cardiovascular risk

Niiranen, Teemu J.

2016-05

Niiranen, T J , Kronholm , E , Rissanen , H , Partinen , M \& Jula , A M 2016 , ' Self-reported obstructive sleep apnea, simple snoring, and various markers of sleep-disordered breathing as predictors of cardiovascular risk ' , Sleep and Breathing , vol. 20 , no. 2 , pp. 589-596 . https://doi.org/10.1007/s11

http://hdl.handle.net/10138/223983

https://doi.org/10.1007/s11325-015-1253-4

publishedVersion

Downloaded from Helda, University of Helsinki institutional repository.

This is an electronic reprint of the original article.

This reprint may differ from the original in pagination and typographic detail.

Please cite the original version. 


\title{
Self-reported obstructive sleep apnea, simple snoring, and various markers of sleep-disordered breathing as predictors of cardiovascular risk
}

\author{
Teemu J. Niiranen ${ }^{1}$ • Erkki Kronholm ${ }^{1}$ • Harri Rissanen ${ }^{1}$ - Markku Partinen ${ }^{2,3}$. \\ Antti M. Jula ${ }^{1}$
}

Received: 4 March 2015 /Revised: 3 August 2015 / Accepted: 3 September 2015 / Published online: 12 September 2015

(C) Springer-Verlag Berlin Heidelberg 2015

\begin{abstract}
Purpose Our objective was to investigate whether selfreported obstructive sleep apnea (OSA), simple snoring, and various markers of sleep-disordered breathing (SDB) are associated with cardiovascular risk.

Methods We examined a representative nationwide cohort of 5177 Finnish adults aged $\geq 30$ years. The participants underwent measurement of traditional cardiovascular risk factors and answered SDB-related questions derived from the Basic Nordic Sleep Questionnaire, which were used to operationalize self-reported OSA. The primary end point was incidence of a cardiovascular event (cardiovascular mortality, non-fatal myocardial infarction, non-fatal stroke, hospitalization for heart failure, or coronary interventions).

Results During a median follow-up of 11.2 years and 52,910 person-years of follow-up, 634 participants suffered a cardiovascular event. In multivariable-adjusted Cox models, selfreported OSA (hazard ratio [HR] 1.34; $95 \%$ confidence interval [CI] $1.04-1.73 ; p=0.03$ ) was an independent predictor of cardiovascular events. Self-reported simple snoring by
\end{abstract}

Teemu J. Niiranen and Erkki Kronholm contributed equally to this work.

Electronic supplementary material The online version of this article (doi:10.1007/s11325-015-1253-4) contains supplementary material, which is available to authorized users.

Teemu J. Niiranen

teemu.niiranen@thl.fi

1 Department of Health, National Institute for Health and Welfare, P.O. Box 57, 20521 Turku, Finland

2 Helsinki Sleep Clinic, Vitalmed Research Centre, Sitratori 3, 00420 Helsinki, Finland

3 Department of Clinical Neurosciences, University of Helsinki, 00100 Helsinki, Finland itself was not associated with future cardiovascular events (HR 0.88 versus non-snorers, $95 \%$ CI 0.75-1.04, $p=0.15$ ). However, among snorers $(n=3152)$, frequent breathing cessations (HR 2.19, $95 \%$ CI 1.26-3.81, $p=0.006$ ) and very loud and irregular snoring (HR 1.82, $95 \%$ CI 1.31-2.54, $p<0.001$ ) were associated with cardiovascular risk.

Conclusions Self-reported OSA and SDB-related snoring variables are associated with cardiovascular risk, whereas simple snoring is not. In clinical practice and in surveys, questions concerning only habitual snoring should be amended with questions focusing on respiratory pauses and snoring stertorousness, which can be used to estimate the risk of OSA and cardiovascular events.

Keywords Sleep $\cdot$ Epidemiology $\cdot$ Sleep apnea $\cdot$ Sleep disorders · Snoring $\cdot$ Questionnaires

\section{Introduction}

The value of self-reported sleep disordered breathing (SDB) as a cardiovascular risk factor remains unclear. Several lines of evidence, including prospective studies, have shown that polysomnographically diagnosed obstructive sleep apnea (OSA) is a causal risk factor for hypertension [1, 2], metabolic syndrome [3], and other cardiovascular manifestations [4] such as stroke [5, 6], coronary artery disease [7], and cardiovascular mortality [8-10]. Polysomnography, however, is a cumbersome and costly examination. In clinical practice, as well as in epidemiological studies, there is a need to use subjective markers of SDB as a first approach in estimating the probability of OSA.

Snoring has been shown to correlate moderately with the apnea-hypopnea [11] and the respiratory disturbance [12] indices. In spite of the somewhat unclear relationships between 
snoring and apnea-hypopnea index or OSA [11], self-reported snoring is often used in epidemiological studies as a marker of OSA and SDB. In these studies, the associations of selfreported snoring with cardiovascular outcomes have been mixed. Several studies have suggested that self-reported snoring is associated with increased risk of incident hypertension [13], metabolic syndrome [14] and type 2 diabetes [15] in women, and with incident cardiovascular disease [16-18] in both genders. However, recent studies with negative results $[19,10,12]$ have initiated a new discussion [20-22] on whether self-reported snoring is truly associated with cardiovascular morbidity and mortality independently of OSA.

One reason underlying these mixed results may be in the differences in phrasing of the snoring-related questions, as snoring can reflect various SDB-related phenomena. Importantly, not all snorers have OSA [11] as portable home recordings have revealed that individuals with no OSA may snore to some extent more than $80 \%$ of the night [12]. Consequently, it would be of interest to study the association between cardiovascular disease and not only simple snoring but also other various self-reported SDB variables, such as the frequency, loudness (stertorousness), and regularity of snoring as well as the presence of respiratory pauses. If the associations between these self-reported SDB variables and cardiovascular outcome differ from each other, this information could be used to improve clinical interviews and epidemiological questionnaires to optimize cardiovascular risk prediction.

The objective of our study was therefore to study if various self-reported SDB variables differ in association with cardiovascular morbidity and mortality in a large-scale, unselected, nationwide prospective cohort. We also examined whether self-reported OSA, operationalized with a series of selfreported SDB variables, would predict cardiovascular outcome. We hypothesized that simple snoring is mainly harmless, but habitual, stertorous snoring in association with breathing cessations would increase the risk of cardiovascular morbidity and mortality.

\section{Methods}

\section{Study population}

The Health 2000 study was a multidisciplinary epidemiological survey, which was carried out in Finland from autumn 2000 to spring 2001 . The study population was a stratified random sample of 8028 subjects drawn from the population register to represent Finnish adults aged $\geq 30$ years. The details of stratification and sampling procedures have been reported previously [23].

Six thousand three hundred fifty-four individuals (79 \%) agreed to participate in the interview and health examination. Subjects who had missing health examination or interview data (office blood pressure, body mass index, smoking status; $n=60)$, missing sleep questionnaire data $(n=698)$, or incomplete laboratory values (fasting cholesterol or glucose level; $n=50$ ) were excluded. Five hundred fifty-four subjects with prior history of cardiovascular disease (see Outcome variables) were identified from the National Hospital Discharge Register backwards until the year 1969 and were also excluded. After removing subjects with $\geq 1$ exclusion factor, 5177 participants were included in the analyses. The study protocol of the Health 2000 Study was approved by the epidemiology ethics committee of the Helsinki and Uusimaa hospital region, and all of the participants gave signed, informed consent.

\section{Flow of the study}

At an initial health interview at the participant's home, information about background, sleep, health, illnesses, and the use of medications were gathered by centrally trained interviewers. The participants underwent a physical examination 1 to 6 weeks later at a local health center. Each participant's height and weight were measured, and fasting blood samples for serum lipids and glucose were taken from the participants. The participants were given the apnea questionnaire after the physical examination, which they were asked to complete and return by mail. Details of the methodology of the project have been published elsewhere [23].

\section{Self-reported variables of SDB and definition of self-reported OSA}

Questions derived from the Basic Nordic Sleep Questionnaire [24] were used to operationalize self-reported OSA as in our earlier studies $[25,26]$. Based on the Third Edition of the International Classification of Sleep Disorders [27], the following logic was applied: If the answer to the question "Do you snore when sleeping? (Ask others if you are not sure)" was "no," self-reported OSA was considered excluded. If the answer was "yes" then the following additional questions were presented: (1) "How often do you snore?"; (2) "What does your snoring sound like? (ask others if needed)"; and (3) "Have you noticed (or have others noticed) respiratory pauses when you sleep?." Self-reported OSA was diagnosed if snoring was frequent (at least during 3-5 nights weekly) and either of the following items was positive: (1) snoring is loud and irregular, with occasional respiratory pauses and/or stertorous breathing, or (2) respiratory pauses with a frequency of at least 1-2 nights weekly. In all other cases, self-reported OSA was considered excluded.

\section{Covariates}

Self-reported sleep duration was determined by asking the subjects, "How many hours do you sleep in $24 \mathrm{~h}$ ?" Responses 
were recorded in full hours. Missing values for sleep duration $(n=100)$ were imputed by age and gender using a regression approach and rounded to the nearest integer. Diabetes mellitus was defined as a fasting serum glucose level of $7.0 \mathrm{mmol} / 1 \mathrm{or}$ greater or the self-reported use of oral hypoglycemic agents or insulin injections, or both. Use of a lipid-lowering medication was defined as a self-reported use of a HMG-CoA reductase inhibitor (statin), fibrate, or nicotinic-acid and its derivatives. Current smoking was defined as a daily use of tobacco products. Body mass index was defined as weight in kilograms divided by squared height in meters.

\section{Outcome variables}

Follow-up data were accumulated until December 31, 2011. Morbid events during the follow-up were identified using the National Hospital Discharge Register, which covers all periods of treatment received in all public and private hospitals in Finland. Mortality data was gathered from the nationwide Causes-of-Death Register. Diagnoses are registered in these registers with codes defined in the 10th revision of the International Classification of Diseases (ICD-10). Cardiovascular diagnoses in these registers, used as described below, have been described and validated in detail previously [28-30].

Acute non-fatal coronary events were identified with ICD10 codes $\mathrm{I} 20$ to $\mathrm{I} 22$ and/or if the participant had underwent percutaneous coronary intervention or coronary artery bypass surgery. For fatal coronary events, ICD-10 codes I20-I25, I46, R96, and R98 were used. Stroke diagnoses in ICD-10 were I60-I61 and I63-I64 (not I63.6). Heart failure diagnoses included ICD-10 codes I50, I110, I130, and I132. Heart failure cases were also identified using the Finnish Drug Reimbursement Register. If the participant was entitled to special reimbursements for the costs of heart failure medications or had purchased furosemide prescribed by a doctor at least three times since 1995 , he/she was considered to have a heart failure.

The primary end point was the combination of cardiovascular mortality, non-fatal myocardial infarction, non-fatal stroke, new onset heart failure, percutaneous coronary intervention, and coronary artery bypass surgery. The secondary endpoints were incident coronary heart disease, stroke, and heart failure. Only the first event was included in the analysis.

\section{Statistical analyses}

Variables were compared using the $\chi^{2}$ test for categorical variables or analysis of variance for continuous variables. We computed direct adjusted Kaplan-Meier survival curves for different groups based on unstratified Cox models [31]. Hazard ratios and $95 \%$ confidence intervals for self-reported OSA and self-reported SDB variables were derived from multivariable-adjusted Cox proportional hazards models. Kaplan-Meier plots were examined to ensure that proportional hazards would be appropriate. The null hypothesis was rejected for values of $p<0.05$. Statistical analyses were performed using SAS 9.2 software (SAS Institute, Cary, NC).

\section{Results}

Table 1 shows the baseline characteristics of the study population. Participants with self-reported OSA were older and had a higher prevalence of smoking and diabetes than participants with no self-reported OSA. In addition, they had higher blood pressure and higher total cholesterol $(p \leq 0.001$ for all). However, there was no difference in self-reported sleep duration between the two groups $(p=0.99)$.

\section{Self-reported OSA and cardiovascular prognosis}

In the overall study population, median follow-up was 11.2 years (5th to 95 th percentile interval, 3.3 to 11.3 years). During 52,910 person-years of follow-up, a fatal or non-fatal cardiovascular endpoint occurred in 634 participants (12.0 per 1000 person-years, Table 2). Five hundred sixty-two of these events occurred in participants with no self-reported OSA ( $n=4782,11.5$ per 1000 person-years) and 72 in participants with self-reported OSA $(n=395,18.7$ per 1000 person-years).

Figure 1 shows the adjusted Kaplan-Meier curve for the composite cardiovascular endpoint by self-reported OSA status. In Cox models adjusted for age, gender, body mass index, smoking status, and sleep duration, there was a significant association of self-reported OSA with incident cardiovascular disease (hazard ratio [HR], $95 \%$ confidence interval [CI] 1.10-1.83], $p=0.007$; Table 2). This association retained its significance after further adjustment for baseline cholesterol level, diabetes mellitus, and blood pressure (HR 1.34, 95 \% CI $1.04-1.73, p=0.03)$. In addition, when abnormal sleep duration ( $<6$ or $\geq 9 \mathrm{~h}$ of sleep) and excessive alcohol use ( $\geq 210 \mathrm{~g}$ of pure alcohol per week) were included as covariates in the model, neither one was significantly associated with the composite endpoint whereas self-reported OSA retained its significance.

We also analyzed the vascular specific endpoints with exclusion of only those with a prior history of that particular endpoint in adjusted Cox models (Table 2). Although there was a non-significant trend for self-reported OSA being a risk factor for coronary events (HR 1.13, $95 \%$ CI $0.80-1.60$, $p=0.49$ ) and heart failure (HR 1.29, $95 \%$ CI $0.90-1.84$, $p=0.17)$, the association was the strongest and significant for stroke (HR 1.65, $95 \%$ CI 1.06-2.57, $p=0.03$ ). 
Table 1 Characteristics by category of self-reported sleep apnea

\begin{tabular}{llll}
\hline & No self-reported OSA & Self-reported OSA & $p$ \\
\hline No. of subjects & 4782 & 395 & \\
Women, $n(\%)$ & $2769(57.9)$ & $92(23.3)$ & $<0.001$ \\
Age, years & $50.7 \pm 14.0$ & $52.3 \pm 10.9$ & $<0.001$ \\
BMI, kg/m ${ }^{2}$ & $26.6 \pm 4.5$ & $28.6 \pm 4.8$ & $<0.001$ \\
Current smoking, $n$ (\%) & $989(20.7)$ & $123(31.1)$ & $<0.001$ \\
Systolic BP, mmHg & $133.3 \pm 20.7$ & $137.2 \pm 19.2$ & $<0.001$ \\
Diastolic BP, mmHg & $81.6 \pm 10.8$ & $85.8 \pm 11.7$ & $<0.001$ \\
Use of antihypertensive medications, $n(\%)$ & $700(14.7)$ & $79(20.0)$ & 0.004 \\
Diabetes mellitus, $n$ (\%) & $203(4.3)$ & $35(8.9)$ & $<0.001$ \\
Cholesterol, mmol/1 & $5.9 \pm 1.1$ & $6.1 \pm 1.1$ & 0.001 \\
Use of lipid-lowering medications, $n(\%)$ & $201(4.2)$ & $26(6.6)$ & 0.03 \\
Sleep duration, $\mathrm{h}$ & $7.5 \pm 1.1$ & $7.5 \pm 1.3 \pm$ & 0.99 \\
\hline
\end{tabular}

Data are reported as mean $\pm \mathrm{SD} . P$ values for differences between groups

$O S A$ obstructive sleep apnea, $B P$ blood pressure, $B M I$ body mass index

\section{Various SDB variables and cardiovascular prognosis}

The independent cardiovascular risk associated with various SDB variables was analyzed with adjusted Kaplan-Meier curves (Fig. 2) and adjusted Cox regression models (Table 3). In contrast to the findings for self-reported OSA, participants who reported simply snoring at least sometimes did not differ in terms of cardiovascular prognosis from those who did not snore at all (Table 3, HR 0.88, $95 \%$ CI 0.75-1.04, $p=0.15$ ). On the other hand, the adjusted survival curves in Fig. 2 showed marked differences in groups by apnea frequency, snoring stertorousness, and snoring frequency among snorers $(n=3152)$. The hazard ratios for cardiovascular events in the category of snoring frequency, apnea frequency, and snoring stertorousness that had the poorest prognosis in the Cox models are reported in Table 3. In these analyses, snoring frequency was not associated with incident cardiovascular events (HR 1.44, $95 \%$ CI 0.94-2.23, $p=0.10$ ) while breathing cessations (HR 2.19, $95 \%$ CI 1.26-3.82, $p=0.006$ ) and snoring stertorousness (HR 1.82, $95 \%$ CI 1.31-2.54, $p<0.001$ ) were. The characteristics of the participants in these categories are reported in Table 4. By and large, participants with increased snoring frequency, apnea frequency, and snoring stertorousness had a poorer cardiovascular risk profile than their counterparts. The hazard ratios for cardiovascular events in all categories of snoring frequency, apnea frequency, and snoring stertorousness are presented in supplementary tables S1, S2, and S3. In general, the cardiovascular risk increased with the degree of self-reported SDB.

\section{Discussion}

Self-reported OSA, operationalized from SDB-related questions, is an independent predictor of cardiovascular events, and especially stroke, in the general population. Out of the

Table 2 Association between self-reported obstructive sleep apnea and incident cardiovascular events

\begin{tabular}{|c|c|c|c|c|c|c|c|c|}
\hline & \multicolumn{2}{|c|}{ Composite events } & \multicolumn{2}{|c|}{ Coronary events } & \multicolumn{2}{|c|}{ Stroke events } & \multicolumn{2}{|c|}{ Heart failure events } \\
\hline & No OSA & OSA & No OSA & OSA & No OSA & OSA & No OSA & OSA \\
\hline No. of participants & 4782 & 395 & 5038 & 422 & 5084 & 437 & 4931 & 416 \\
\hline No. of events, $n(\%)$ & $562(11.8)$ & $72(18.2)$ & $325(6.5)$ & $39(7.7)$ & $171(3.4)$ & $24(5.5)$ & $339(6.9)$ & $36(8.7)$ \\
\hline Model 1, HR (95 \% CI) & 1.00 (ref) & $1.42(1.10-1.83)^{* *}$ & 1.00 (ref) & $1.25(0.88-1.75)$ & 1.00 (ref) & $1.69(1.09-2.63)^{*}$ & 1.00 (ref) & $1.34(0.94-1.91)$ \\
\hline Model 2, HR (95 \% CI) & 1.00 (ref) & $1.33(1.03-1.72)^{*}$ & 1.00 (ref) & $1.14(0.81-1.61)$ & 1.00 (ref) & $1.66(1.07-2.59)^{*}$ & 1.00 (ref) & $1.29(0.90-1.84)$ \\
\hline Model 3, HR (95 \% CI) & 1.00 (ref) & $1.34(1.04-1.73)^{*}$ & 1.00 (ref) & $1.13(0.80-1.60)$ & 1.00 (ref) & $1.65(1.06-2.57)^{*}$ & 1.00 (ref) & $1.29(0.90-1.84)$ \\
\hline
\end{tabular}

Covariates in model 1: age, gender, body mass index, smoking, sleep duration; model 2: model $1+$ total cholesterol, lipid-lowering medications, diabetes; model 3: model $2+$ systolic blood pressure, use of antihypertensive medications. Participants with prior history of the particular endpoint were excluded from the analysis

$O S A$ obstructive sleep apnea, $H R$ hazard ratio, $C I$ confidence interval

${ }^{*} p<0.05,{ }^{* *} p<0.01$ 


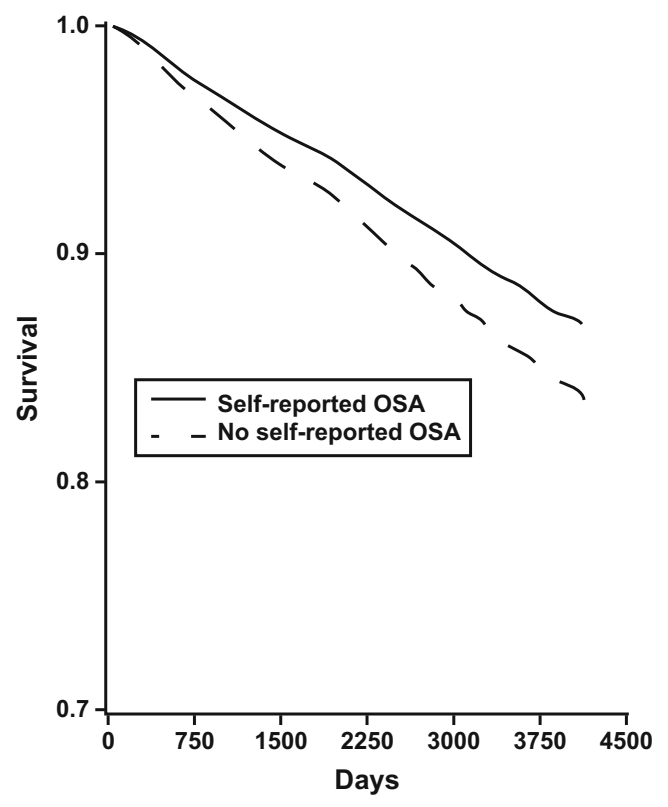

Fig. 1 Adjusted Kaplan-Meier survival curves for composite cardiovascular events of participants with or without self-reported sleep apnea. $O S A$ obstructive sleep apnea

specific characteristics of snoring, increased breathing cessations and snoring stertorousness seem to be associated with cardiovascular outcome whereas self-reported simple snoring is not.

In addition to highlighting the dangers of OSA, our study reached the same conclusion as two previous studies by Marin et al. and Davis et al. [19, 10] —no association could be found between simple snoring and increased cardiovascular risk. Our results are also in line with a recent study with portable home audio recording of snoring, which suggested that snoring as itself is not associated with future cardiovascular events or mortality [12]. On the other hand, some previous studies have found habitual snoring to be associated with a modest increase of incident coronary heart disease and stroke after adjustment for pertinent risk factors among women [18, 16] and men [17]. This discrepancy in results from various studies could be caused by differences in mean age of the study participants or in operationalizations of (self-reported) snoring.

We suggest that the underlying mechanism behind the associations of self-reported variables for SDB and cardiovascular events is OSA and/or other consequences of habitual snoring rather than simple snoring in itself. The role of intermittent hypoxia, usually measured with the apnea-hypopnea index (AHI, the number of periods of obstructed breathing per hour of sleep), has been shown to be independently associated with cardiovascular morbidity and mortality [5-10]. Repetitive obstructive apneas have been shown to expose the heart and circulation to a cascade of noxious stimuli, such as negative intrathoracic pressure, autonomic dysregulation, platelet activation, oxidative stress, and endothelial dysfunction, that may initiate or contribute to the progression of cardiovascular disorders [32]. Most likely self-reported breathing cessations and stertorous snoring are markers of an increased AHI, associated with a poor cardiovascular prognosis. On the other hand, simple snoring has been shown to have only a weak association with AHI [11].

Given the expenses and hurdle of full-night polysomnography, it is clear that the use of self-reported markers of OSA and SDB will continue for screening purposes in clinical use and epidemiological studies. The results of our study highlight the need for questionnaire items
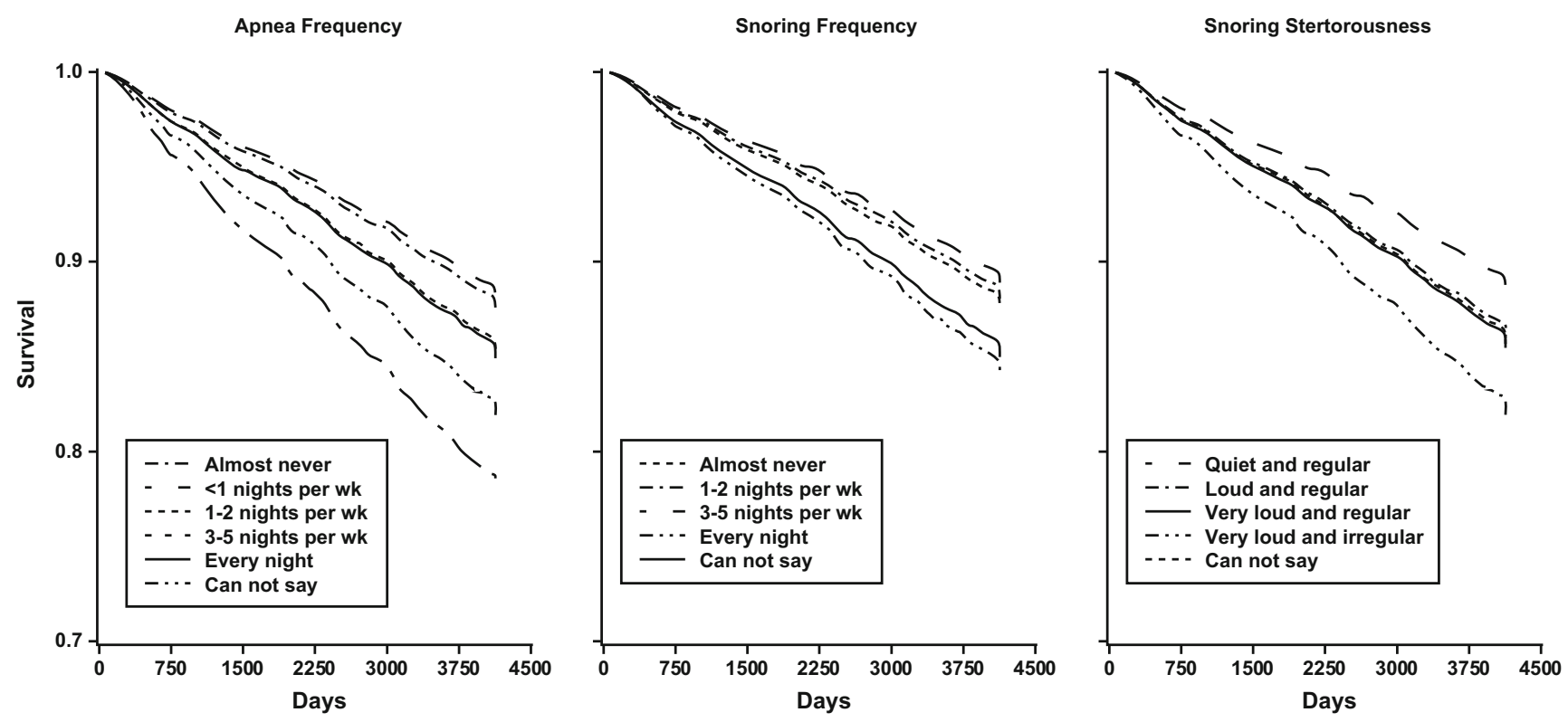

Fig. 2 Adjusted Kaplan-Meier survival curves for composite cardiovascular events among snorers $(n=3152)$ by apnea frequency, snoring stertorousness, and snoring frequency 
Table 3 Various self-reported variables of sleep-disordered breathing as predictors of cardiovascular events

\begin{tabular}{|c|c|c|c|c|c|c|c|c|}
\hline & \multicolumn{2}{|c|}{ Simple snoring } & \multicolumn{2}{|c|}{ Snoring frequency } & \multicolumn{2}{|c|}{ Apnea frequency } & \multicolumn{2}{|c|}{ Snoring stertorousness } \\
\hline & No & Yes & $\begin{array}{l}\text { Almost } \\
\text { never }\end{array}$ & Every night & $\begin{array}{l}\text { Almost } \\
\text { never }\end{array}$ & $\begin{array}{l}3-5 \text { nights per } \\
\text { week }\end{array}$ & $\begin{array}{l}\text { Quiet and } \\
\text { regular }\end{array}$ & $\begin{array}{l}\text { Very loud and } \\
\text { irregular }\end{array}$ \\
\hline No. of participants & 2025 & 3152 & 425 & 924 & 1608 & 62 & 937 & 412 \\
\hline No. of events & 243 & 387 & 25 & 148 & 139 & 14 & 76 & 72 \\
\hline Crude HR (95 \% CI) & 1.00 (ref) & $1.02(0.87-1.20)$ & 1.00 (ref) & $2.91(1.90-4.44)^{* *}$ & 1.00 (ref) & $2.82(1.63-4.88)^{* *}$ & 1.00 (ref) & $2.30(1.66-3.17)^{* *}$ \\
\hline Adjusted HR (95 \% CI) & 1.00 (ref) & $0.88(0.75-1.04)$ & 1.00 (ref) & $1.44(0.94-2.23)$ & 1.00 (ref) & $2.19(1.26-3.81)^{*}$ & 1.00 (ref) & $1.82(1.31-2.54)^{* *}$ \\
\hline
\end{tabular}

Age, gender, body mass index, smoking, sleep duration, total cholesterol, lipid-lowering medications, diabetes, systolic blood pressure, and use of antihypertensive medications were included as covariates in the adjusted models

$H R$ hazard ratio, $C I$ confidence interval

${ }^{*} p<0.05,{ }^{* *} p<0.001$

assessing OSA and SDB to be formulated in a way that maximally captures the information indicating the presence of OSA and increased cardiovascular risk rather than snoring in general. Our results show that, instead of focusing on only snoring in itself, more attention should be focused on snoring stertorousness and frequency of respiratory pauses among snorers.

A strength of this study is the large sample representative of the nationwide adult population living in Finland. Another strength is the relatively long follow-up of the cohort and nationwide coverage of the cardiovascular events based on high-quality register data. However, the small number of events among participants with self-reported OSA prevents from drawing definite conclusions. Lung disease and depression were not included as covariates in the Cox models which could also be a limitation of this study. Furthermore, our data are self-reported and we do not have polysomnographic validation of the self-reported markers of SDB.

Taken together, our results suggest that self-reported markers of SDB such as habitual snoring, breathing cessations, and stertorous and irregular sound are dimensions of snoring which are associated with incident cardiovascular events. Occasional snoring, i.e., simple snoring in general, is not associated with increased cardiovascular risk and may not be a clinically meaningful target for intervention. In clinical practice and in surveys, screening of individuals for suspected OSA should concentrate on the characteristics of snoring. Our study highlights the need to examine the association between

Table 4 Characteristics by categories of simple snoring or by category of snoring frequency, apnea frequency, and snoring stertorousness among snorers

\begin{tabular}{|c|c|c|c|c|c|c|c|c|}
\hline & \multicolumn{2}{|c|}{ Simple snoring } & \multicolumn{2}{|c|}{ Snoring frequency } & \multicolumn{2}{|c|}{ Apnea frequency } & \multicolumn{2}{|c|}{ Snoring stertorousness } \\
\hline & No & Yes & $\begin{array}{l}\text { Almost } \\
\text { never }\end{array}$ & Every night & $\begin{array}{l}\text { Almost } \\
\text { never }\end{array}$ & $\begin{array}{l}3-5 \text { nights } \\
\text { per week }\end{array}$ & $\begin{array}{l}\text { Quiet and } \\
\text { regular }\end{array}$ & $\begin{array}{l}\text { Very loud and } \\
\text { irregular }\end{array}$ \\
\hline No. of subjects & 2025 & 3152 & 425 & 924 & 1608 & 62 & 937 & 412 \\
\hline Women, $n(\%)$ & $1336(66.0)$ & $1525(48.4)^{* *}$ & $260(61.0)$ & $341(36.9)^{* *}$ & $825(51.3)$ & $13(21.0)^{* *}$ & $559(59.7)$ & $108(26.2)^{* *}$ \\
\hline Age, years & $49.8 \pm 15.0$ & $51.4 \pm 12.8^{* *}$ & $46.7 \pm 11.8$ & $52.1 \pm 51.5^{* *}$ & $49.4 \pm 12.4$ & $51.9 \pm 9.4$ & $49.7 \pm 13.2$ & $52.1 \pm 11.0^{* *}$ \\
\hline BMI, $\mathrm{kg} / \mathrm{m}^{2}$ & $25.6 \pm 4.2$ & $27.5 \pm 4.6^{* *}$ & $25.9 \pm 4.1$ & $28.7 \pm 4.7^{* *}$ & $27.0 \pm 4.4$ & $28.3 \pm 4.0$ & $26.6 \pm 4.2$ & $28.3 \pm 4.9^{* *}$ \\
\hline Current smoking, $n(\%)$ & $368(18.2)$ & $744(23.6)^{* *}$ & $74(17.4)$ & $129(19.4)^{* *}$ & $333(20.7)$ & $13(21.0)$ & 177 (18.9) & $130(31.6) * * *$ \\
\hline Systolic BP, mmHg & $131.5 \pm 21.1$ & $134.8 \pm 20.0 * *$ & $129.2 \pm 19.2$ & $136.0 \pm 19.0^{* *}$ & $132.8 \pm 20.1$ & $139.3 \pm 19.8^{*}$ & $132.0 \pm 19.8$ & $136.0 \pm 19.0 * *$ \\
\hline Diastolic BP, mmHg & $79.8 \pm 10.9$ & $83.3 \pm 10.7^{* *}$ & $80.8 \pm 10.4$ & $84.7 \pm 10.9 * *$ & $82.7 \pm 10.4$ & $87.8 \pm 9.6^{* *}$ & $81.4 \pm 10.3$ & $85.4 \pm 11.5^{* *}$ \\
\hline AH use, $n(\%)$ & $267(13.2)$ & $512(16.2)^{*}$ & $47(11.0)$ & $168(18.2)^{* *}$ & $214(13.3)$ & $11(17.7)$ & $118(12.6)$ & $81(19.7) * *$ \\
\hline Diabetes mellitus, $n(\%)$ & $82(4.1)$ & $156(5.0)$ & $14(3.3)$ & $63(6.8)^{*}$ & $62(3.9)$ & $7(11.3)^{*}$ & $31(3.3)$ & $31(7.5)^{* *}$ \\
\hline Cholesterol, mmol/1 & $5.8 \pm 1.1$ & $6.0 \pm 1.1 * *$ & $5.9 \pm 1.1$ & $6.1 \pm 1.1 * *$ & $6.0 \pm 1.1$ & $6.2 \pm 1.2$ & $6.0 \pm 1.1$ & $6.1 \pm 1.1^{*}$ \\
\hline Use of statins, $n(\%)$ & $84(4.2)$ & $143(4.5)$ & $10(2.4)$ & $55(6.0)^{*}$ & $59(3.7)$ & $3(4.8)$ & $35(3.7)$ & $21(5.1)$ \\
\hline Sleep duration, $\mathrm{h}$ & $7.5 \pm 1.1$ & $7.4 \pm 1.1 *$ & $7.5 \pm 1.0$ & $7.4 \pm 1.1$ & $7.4 \pm 1.0$ & $7.4 \pm 1.1 * *$ & $7.5 \pm 1.0$ & $7.4 \pm 1.2$ \\
\hline
\end{tabular}

Data are reported as mean $\pm \mathrm{SD}$

$A H$ antihypertensive, $B P$ blood pressure, $B M I$ body mass index

${ }^{*} p<0.05,{ }^{* *} p<0.001$ - values for differences between groups 
characteristics of snoring and cardiovascular disease in more observational settings and with more objective and precise markers of SDB such as polysomnography which would help establish true causality.

Acknowledgments The project organization created for the study involved the Finnish Centre for Pensions, the Social Insurance Institution, the National Public Health Institute, the Local Government Pensions Institution, the National Research and Development Centre for Welfare and Health, the Finnish Dental Society and the Finnish Dental Association, Statistics Finland, the Finnish Work Environment Fund, the Finnish Institute for Occupational Health, the UKK Institute for Health Promotion, the State Pensions Office, and the State Work Environment Fund.

\section{Compliance with ethical standards}

Ethical standards All participants of the study gave their informed consent prior to inclusion in the study.

Conflict of interest The authors declare that they have no competing interests.

\section{References}

1. Phillips CL, O'Driscoll DM (2013) Hypertension and obstructive sleep apnea. Nat Sci Sleep 5:43-52

2. Peppard PE, Young T, Palta M, Skatrud J (2000) Prospective study of the association between sleep-disordered breathing and hypertension. N Engl J Med 342:1378-1384

3. Lam JCM, Ip MSM (2007) An update on obstructive seep apnea and the metabolic syndrome. Curr Opin Pulm Med $13: 484-489$

4. Dong JY, Zhang YH, Qin LQ (2013) Obstructive sleep apnea and cardiovascular risk: meta-analysis of prospective cohort studies. Atherosclerosis 229:489-495

5. Yaggi HK, Concato J, Kernan WN, Lichtman JH, Brass LM, Mohsenin V (2005) Obstructive sleep apnea as a risk factor for stroke and death. N Engl J Med 353:2034-2041

6. Redline S, Yenokyan G, Gottlieb DJ, Shahar E, O'Connor GT, Resnick HE, Diener-West M, Sanders MH, Wolf PA, Geraghty EM, Ali T, Lebowitz M, Punjabi NM (2010) Obstructive sleep apnea-hypopnea and incident stroke: the sleep heart health study. Am J Respir Crit Care Med 182:269-277

7. Peker Y, Kraiczi H, Hedner J, Loth S, Johansson A, Bende $M$ (1999) An independent association between obstructive sleep apnoea and coronary artery disease. Eur Resp J 14: 179-184

8. Young T, Finn L, Peppard PE, Szklo-Coxe M, Austin D, Nieto J, Stubbs R, Hla KM (2008) Sleep disordered breathing and mortality: eighteen-year follow-up of the Wisconsin sleep cohort. Sleep 31: 1071-1078

9. Punjabi NM, Caffo BS, Goodwin JL, Gottlieb DJ, Newman AB, O'Connor GT, Rapoport DM, Redline S, Resnick HE, Robbins JA, Shahar E, Unruh ML, Samet JM (2009) Sleep-disordered breathing and mortality: a prospective cohort study. PLoS Med. doi:10.1371/ journal.pmed.1000132

10. Marin JM, Carrizo SJ, Vicente E, Agusti AGN (2005) Longterm cardiovascular outcomes in men with obstructive sleep apnoea-hypopnoea with or without treatment with continuous positive airway pressure: an observational study. Lancet 365 : 1046-1053

11. Bearpark H, Elliott L, Grunstein R, Cullen S, Schneider H, Althaus W, Sullivan C (1995) Snoring and sleep apnea. A population study in Australian men. Am J Respir Crit Care Med 151:1459-1465

12. Marshall NS, Wong KKH, Cullen SRJ, Knuiman MW, Grunstein RR (2012) Snoring is not associated with all-cause mortality, incident cardiovascular disease, or stroke in the Busselton Health Study. Sleep 35:1235-1240

13. Knutson KL, Van Cauter E, Rathouz PJ, Yan LL, Hulley SB, Liu K, Lauderdale DS (2009) Association between sleep and blood pressure in midlife: the CARDIA sleep study. Arch Intern Med 169: 1055-1061

14. Leineweber C, Kecklund G, Akerstedt T, Janszky I, Orth-Gomer K (2003) Snoring and the metabolic syndrome in women. Sleep Med 4:531-536

15. Al-Delaimy WK, Manson JE, Willett WC, Stampfer MJ, Hu FB (2002) Snoring as a risk factor for type II diabetes mellitus: a prospective study. Am J Epidemiol 155:387-393

16. Hu FB, Willett WC, Manson JAE, Colditz GA, Rimm EB, Speizer FE, Hennekens CH, Stampfer MJ (2000) Snoring and risk of cardiovascular disease in women. J Am Coll Cardiol 35:308-313

17. Koskenvuo M, Kaprio J, Telakivi T, Partinen M, Heikkilä K, Sarna S (1987) Snoring as a risk factor for ischaemic heart disease and stroke in men. Br Med J 294:16-19

18. Sands M, Loucks EB, Lu B, Carskadon MA, Sharkey K, Stefanick M, Ockene J, Shah N, Hairston KG, Robinson J, Limacher M, Hale L, Eaton CB (2013) Self-reported snoring and risk of cardiovascular disease among postmenopausal women (from the Women's Health Initiative). Am J Cardiol 111:540-546

19. Davies DP, Rodgers H, Walshaw D, James OFW, Gibson GJ (2003) Snoring, daytime sleepiness and stroke: a case-control study of first-ever stroke. J Sleep Res 12:313-318

20. Marshall NS, Wong KKH, Cullen SRJ, Knuiman MW, Grunstein RR (2013) The burden of proof lies with the prosecution: is snoring guilty? Sleep 36:615

21. Kezirian EJ (2013) Snoring without OSA and health consequences: the jury is still out. Sleep 36:613

22. Rice TB (2012) Noise in the signal or bad vibrations? Sleep 35: 1193-1194

23. Heistaro S (2008) Methodology report. Health 2000 Survey. National Public Health Institute, Helsinki

24. Partinen M, Gislason T (1995) Basic Nordic Sleep Questionnaire (BNSQ): a quantitated measure of subjective sleep complaints. J Sleep Res 4:150-155

25. Fogelholm M, Kronholm E, Kukkonen-Harjula K, Partonen T, Partinen M, Härmä M (2007) Sleep-related disturbances and physical inactivity are independently associated with obesity in adults. Int J Obes 31:1713-1721

26. Johansson JK, Kronholm E, Jula A (2011) Variability of home-measured blood pressure and heart rate: associations with self-reported insomnia and sleep duration. J Hypertens 29:1897-1905

27. International Classification of Sleep Disorders, 3rd ed. (2014) American Academy of Sleep Medicine, Chicago

28. Pajunen P, Koukkunen H, Ketonen M, Jerkkola T, Immonen-Räiha P, Karja-Koskenkari PK, Mahonen M, Niemela M, Kuulasmaa K, Palomäki P, Mustonen J, Lehtonen A, Arstila M, Vuorenmaa T, Lehto S, Miettinen H, Torppa J, Tuomilehto J, Kesaniemi YA, Pyorälä K, Salomaa V (2005) The validity of the Finnish hospital discharge register and causes of death register data on coronary heart disease. Eur J Cardiovasc Prev Rehabil 12:132-137 
29. Mähönen M, Jula A, Harald K, Antikainen R, Tuomilehto J, Zeller T, Blankenberg S, Salomaa V (2013) The validity of heart failure diagnoses obtained from administrative registers. Eur J Prev Cardiol 20:254-259

30. Tolonen H, Salomaa V, Torppa J, Sivenius J, Immonen-Räiha P, Lehtonen A (2007) The validation of the Finnish hospital discharge register and causes of death register data on stroke diagnoses. Eur J Cardiovasc Prev Rehabil 14:380-385
31. Zhang X, Loberiza FR, Klein JP, Zhang M-J (2007) A SAS macro for estimation of direct adjusted survival curves based on a stratified Cox regression model. Comput Methods Prog Biomed 88:95-101

32. Kasai T, Floras JS, Bradley TD (2012) Sleep apnea and cardiovascular disease: a bidirectional relationship. Circulation 126:14951510 